\title{
Capture and release of a conditional state of a cavity QED system by quantum feedback.
}

\author{
W. P. Smith ${ }^{1}$, J. E. Reiner ${ }^{1}$, L. A. Orozco ${ }^{1}$, S. Kuhr ${ }^{2}$, and H. M. Wiseman ${ }^{3}$ \\ ${ }^{1}$ Dept. Physics and Astronomy, SUNY Stony Brook, Stony Brook NY 11794-3800, USA. \\ 2 Institut für Angewandte Physik, Universität Bonn, Wegelerstr. 8, D-53115 Bonn, Germany. \\ ${ }^{3}$ Center for Quantum Dynamics, School of Science, Griffith University, Brisbane, Queensland 4111, Australia.
}

(February 1, 2008)

\begin{abstract}
Detection of a single photon escaping an optical cavity QED system prepares a non-classical state of the electromagnetic field. The evolution of the state can be modified by changing the drive of the cavity. For the appropriate feedback, the conditional state can be captured (stabilized) and then released. This is observed by a conditional intensity measurement that shows suppression of vacuum Rabi oscillations for the length of the feedback pulse and their subsequent return.
\end{abstract}

Feedback control of quantum systems was first studied about fifteen years ago [1] 3], in the field of quantum optics. In these approaches, the feedback could be understood in an essentially classical way, with quantum field theory entering only to dictate the magnitude of the fluctuations. This is possible if fluctuations are small compared to the mean fields being detected. More recently, a different approach to quantum optical feedback has been developed [4.5], based on quantum trajectories [6] 8], which specify the stochastic evolution of a quantum state conditioned on continuous monitoring (such as by photodetection). This theory allows the treatment of feedback in the deep quantum regime, where quantum fluctuations are not small compared to the mean. It is also arguably the best way to approach feedback, as the conditioned state by definition comprises all of the knowledge of the experimenter on which feedback could be based [9, 10].

So far, experiments in quantum feedback, such as Refs. [1]11 15], have all been in the regime of small fluctuations [16]. Cavity QED is able to explore the opposite regime, where fluctuations in the conditional state are large. Furthermore, using the theory of quantum trajectories, Carmichael and coworkers [17,18] showed that such conditional quantum fluctuations are intrinsically related to the production of squeezing and antibunching. In this letter we present experimental results for the application of feedback in this regime. Following a photodetection, the conditioned quantum state of the system is $\left|\psi_{\mathrm{c}}(\tau)\right\rangle$. Given our knowledge of this evolution, we can, for certain times $\tau$, change the parameters of the system dynamics so as to capture the system in that conditioned state. When the parameters are later restored to their usual values, the released system state resumes its interrupted evolution. This directly demonstrates both the reality of the conditioned state and its usefulness for quantum feedback.

A Cavity quantum electrodynamical (QED) system consists of a single mode of the electromagnetic field of a cavity interacting with one or a collection of $N$ two-level atoms [19]. Microwave Cavity QED systems have been used recently to prepare multiparticle entanglement [20], and to produce photon number states of the electromagnetic field 21. Operated at optical frequencies, cavity QED systems can now trap single atoms in the electric field of the cavity when its average occupation is about one photon [22, 23]. The system size and dynamics are characterized by two dimensionless numbers: The saturation photon number $n_{0}$ and the single atom cooperativity $C_{1}$. They scale the influence of a photon and the influence of an atom in cavity QED. These two numbers relate the reversible dipole coupling of a single atom with the cavity mode $(g)$ with the irreversible coupling to the reservoirs through cavity $(\kappa)$ and atomic radiative $(\gamma)$ decays: $C_{1}=g^{2} / \kappa \gamma$ and $n_{0}=\gamma^{2} / 3 g^{2}$. The strong coupling regime of cavity QED requires $n_{0} \leq 1$, and $C_{1} \geq 1$. Strictly speaking, the coupling constant $(g)$ is spatially dependent and together with the $N$ moving atoms may be described by effective constants.

With weak driving, the system can be accurately modelled as having either zero, one, or two excitations of the coupled normal modes of the field and the atoms. In this regime, photodetections are very infrequent and the state before a detection can be taken to be the steady state, which is almost pure:

$$
\begin{aligned}
\left|\psi_{\mathrm{ss}}\right\rangle= & |0, G\rangle+\lambda\left(|1, G\rangle-\frac{2 g \sqrt{N}}{\gamma}|0, E\rangle\right) \\
& +\lambda^{2}\left(\zeta_{0} \frac{1}{\sqrt{2}}|2, G\rangle-\theta_{0} \frac{2 g \sqrt{N}}{\gamma}|1, E\rangle\right)+\cdots
\end{aligned}
$$

Here $|n, G\rangle$ represents $n$ photons with all $(N)$ atoms in their ground state, $|n, E\rangle$ represents $n$ photons with one atom in the excited state with the rest $(N-1)$ in their ground state. The small parameter is $\lambda=\langle\hat{a}\rangle=$ $\epsilon / \kappa\left(1+C_{1} N\right)$, which depends on the input driving field $\epsilon$, while $\zeta_{0}$ and $\theta_{0}$ are coefficients of order unity for the two-excitation component of the state that can give 
rise to a photon detection, and depend on $g, \kappa$, and $\gamma$ 224,25. After the photodetection occurs $\left|\psi_{\mathrm{ss}}\right\rangle$ collapses to $\hat{a}\left|\psi_{\mathrm{ss}}\right\rangle / \sqrt{\left\langle\hat{a}^{\dagger} \hat{a}\right\rangle_{\mathrm{ss}}}$. This evolves as the conditioned state:

$$
\begin{aligned}
\left|\psi_{\mathrm{c}}(\tau)\right\rangle= & |0, G\rangle+\lambda\left(\zeta(\tau)|1, G\rangle-\theta(\tau) \frac{2 g \sqrt{N}}{\gamma}|0, E\rangle\right) \\
& +O\left(\lambda^{2}\right)
\end{aligned}
$$

This is different from the initial state because $\zeta$ (the "field" evolution) and $\theta$ (the "atomic polarization" evolution) oscillate coherently at the vacuum Rabi frequency $(g \sqrt{N})$ over time as the system re-equilibrates exchanging energy between the atomic polarization and the cavity field 24,25].

If we choose a time $\tau=T$ for Eq. (2) such that $\zeta(T)=\theta(T)$ then, to order $\lambda$ we obtain

$$
\left|\psi_{\mathrm{c}}(T)\right\rangle \simeq|0, G\rangle+\lambda^{\prime}\left(|1, G\rangle-\frac{2 g \sqrt{N}}{\gamma}|0, E\rangle\right),
$$

This is of the form of $\left|\psi_{\text {ss }}\right\rangle$ in Eq. (1) but with a different mean field $\lambda^{\prime}=\zeta(T) \lambda$. This conditional state can be stabilized if, at time $T$, we change the driving amplitude by a factor $\zeta(T)$. Given the almost $90^{\circ}$ out of phase oscillations between the field $(\zeta)$ and the atomic polarization $(\theta)$ [25] the time $T$ is close to the time when the field is crossing zero.

Conditional quantum states such as Eq. 2 can be measured using high order quantum optical correlations [18,26. When the light transmitted through the cavity (with annihilation operator $\hat{a}$ ) is split the photons enter two detectors. The normalized correlation function of the two photocurrents is the time-and normally ordered average

$$
\begin{aligned}
g^{(2)}(\tau) & =\frac{\left\langle\hat{a}^{\dagger}(t) \hat{a}^{\dagger}(t+\tau) \hat{a}(t+\tau) \hat{a}(t)\right\rangle_{\mathrm{ss}}}{\left\langle\hat{a}^{\dagger}(t) \hat{a}(t)\right\rangle_{\mathrm{ss}}^{2}} \\
& =\frac{\langle\hat{n}(t+\tau)\rangle_{\mathrm{c}}}{\langle\hat{n}(t)\rangle_{\mathrm{ss}}},
\end{aligned}
$$

where $\hat{n}=\hat{a}^{\dagger} \hat{a}$, and c means "conditioned on a detection at time $t$ in steady state". If a detection at one detector is used to trigger a feedback pulse on the system, the correlation function will no longer be time symmetric. However, for $\tau>0$ the expression (4) still measures the conditional state in the presence of feedback:

$$
g^{(2)}(\tau) \simeq \frac{\left|\left\langle 1, G \mid \psi_{\mathrm{c}}(\tau)\right\rangle\right|^{2}}{\mid\left\langle 1, G\left|\psi_{\mathrm{ss}}\right|^{2}\right.}=[\zeta(\tau)]^{2}
$$

Fig. 1 shows the conditional evolution of the state of the cavity QED system, as given by Eq. 5 . We start with the quantum theory valid for $N$ two level atoms identically coupled to the cavity in the weak field regime [24]. We find $g_{\text {eff }}<g$ and $N_{\text {eff }}$ 28,29] using our experimentally determined values for $g^{(2)}(0)$ such that $g \sqrt{N}=g_{\text {eff }} \sqrt{N_{\text {eff }}}$.
All broadening effects are incorporated by the modification of the atomic decay rate, $\gamma \rightarrow \gamma^{\prime}$. We numerically solve the time evolution with the driving step incorporated. This simplified approach agrees with our previous work for $g^{(2)}(\tau)$ [27]. The dashed line is the free evolution of the system, and shows the time symmetry of the correlation function. The application of a feedback pulse at time $T$ alters the evolution of the system. The continuous lines shows the evolution when the step change in the driving intensity $\epsilon$ satisfies the conditions necessary to reach a new steady state described by Eq. 3. The parameters of the calculation are those of our experiment: $g \sqrt{N} / 2 \pi=37.3 \mathrm{MHz}, \gamma^{\prime} / 2 \pi=9.1 \mathrm{MHz}$ and $\kappa / 2 \pi=3.7$ MHz. The change in the intensity is small $(0.2 \%)$ and here we assume that its rise and fall are instantaneous. The new state reached by the system after the change of driving intensity no longer shows the vacuum Rabi oscillations and instead has a new value for the steady state slightly lower than the original one. The duration of the pulse that changes the steady state is finite and our model shows the reappearance of the oscillation delayed by the length of the pulse.

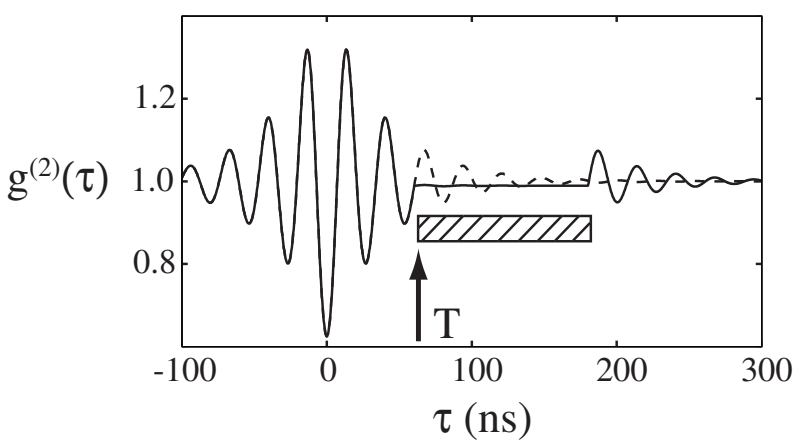

FIG. 1. Conditional evolution of cavity QED system with (continuous line) and without (dashed line) feedback. The shaded rectangle region corresponds to an instantaneous step down of $0.2 \%$ of the driving intensity applied at time $T$.

Our cavity QED apparatus, described in detail in Ref. 27], consists of the cavity, the atomic beam, an excitation laser, and the detector system. Two high reflectivity curved mirrors (input transmission mirror 10 ppm, output transmission mirror $285 \mathrm{ppm}$, and separation $l=880 \mu \mathrm{m}$ ) form the optical cavity (waist of the $\mathrm{TEM}_{00}$ mode $w_{0}=34 \mu \mathrm{m}$ ). A Pound-Drever-Hall stabilization technique keeps the cavity locked to the appropriate atomic resonance. An effusive oven produces a thermal $(440 \mathrm{~K})$ beam of $\mathrm{Rb}$ atoms with an angular spread of 2.8 mrads at the cavity mode. A laser beam intersects the atomic beam before the atoms enter the cavity in a region with 2.5 Gauss uniform magnetic field. It optically pumps all the ${ }^{85} \mathrm{Rb}$ atoms of the $F=3$ ground state the magnetic sublevel $F=3, m_{F}=3$. The three rates that characterize our cavity QED system are $(g, \kappa, \gamma / 2) / 2 \pi=(5.1,3.7,3.0) \mathrm{MHz}$. 


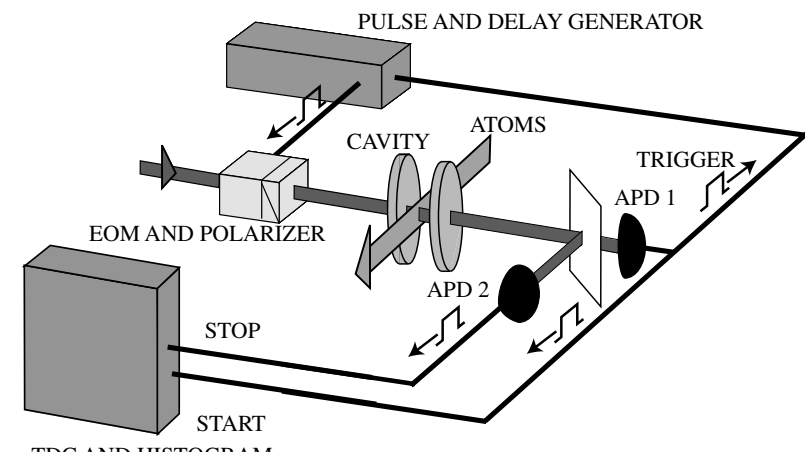

TDC AND HISTOGRAM

FIG. 2. Simplified diagram of the experimental setup. The output of the cavity QED system passes through a beam splitter (entrance of an intensity correlator) such that the detection of a photon at the "start" avalanche photodiode (APD) also triggers a change in the driving intensity trough a pulse that drives an electro-optical modulator EOM in front of a polarizer. A histogram of the delays between the "start" and "stop" gives the conditional evolution of the intensity

Fig. 2 shows a schematic of our apparatus. Light from a Ti:Sapph, locked to the $5 S_{1 / 2}, F=3 \rightarrow 5 P_{3 / 2}, F=4$ transition of ${ }^{85} \mathrm{Rb}$ at $780 \mathrm{~nm}$, drives the cavity QED system. The signal escaping the cavity creates photodetections at the "Start" and "Stop" avalanche photodiodes (APD). The output pulse of the "Start" detector is split and one part sent to the start channel of the correlator [time to digital converter (TDC) with 0.5 ns per bin, histograming memory, and computer] while the other goes to a variable time delay, and after pulse shaping and lengthening, drives an Electro Optical Modulator (EOM) in front of a polarizer to produce a pulse of 8 ns risetime and $120 \mathrm{~ns}$ length in the driving intensity of the cavity. The delay between the detection of a photon at APD1 and the arrival of the pulse at the cavity can be as short as $45 \mathrm{~ns}$. The other APD sends its pulses to the correlator to stop the TDC that measures the time interval between the two events.

We operate the cavity QED system in a non-classical regime where the size of the vacuum Rabi oscillations is large enough to permit their rapid identification during data taking. We begin by measuring the antibunched second order correlation function of the intensity escaping our cavity QED system. We then apply the step change in the driving intensity at time $T$ to fulfill the conditions of Eq. (3) and obtain a new steady state.

Fig. 3 shows measurements of the correlation function in the absence (i) and presence (ii, iii) of feedback. Traces $\mathrm{i}$ and ii have the same oscillating frequency while for trace iii we have a smaller number of atoms. $\tau^{*}$ marks the position where the oscillation we want to suppress reaches a maximum. The steady state for $\tau$ large corresponds to an intracavity intensity of $n / n_{0}=0.07$. Fig. 3 ii shows the correlation function with step down feedback (- 2.6 \%) for $120 \mathrm{~ns}$, beginning at $\tau=T=57 \mathrm{~ns}$, when the oscillation crosses unity and is growing. The oscillation that has a maximum in trace $\mathrm{i}$ at the point marked by $\tau^{*}$ has disappeared, the steady state is lower that marked by the dashed line, and the oscillation reappears after the pulse is turned off with approximately the same size of the amplitude as the suppressed one. Trace iii shows step up feedback $(+3.9 \%)$ at $T=46 \mathrm{~ns}$ when the phase is opposite from trace ii.

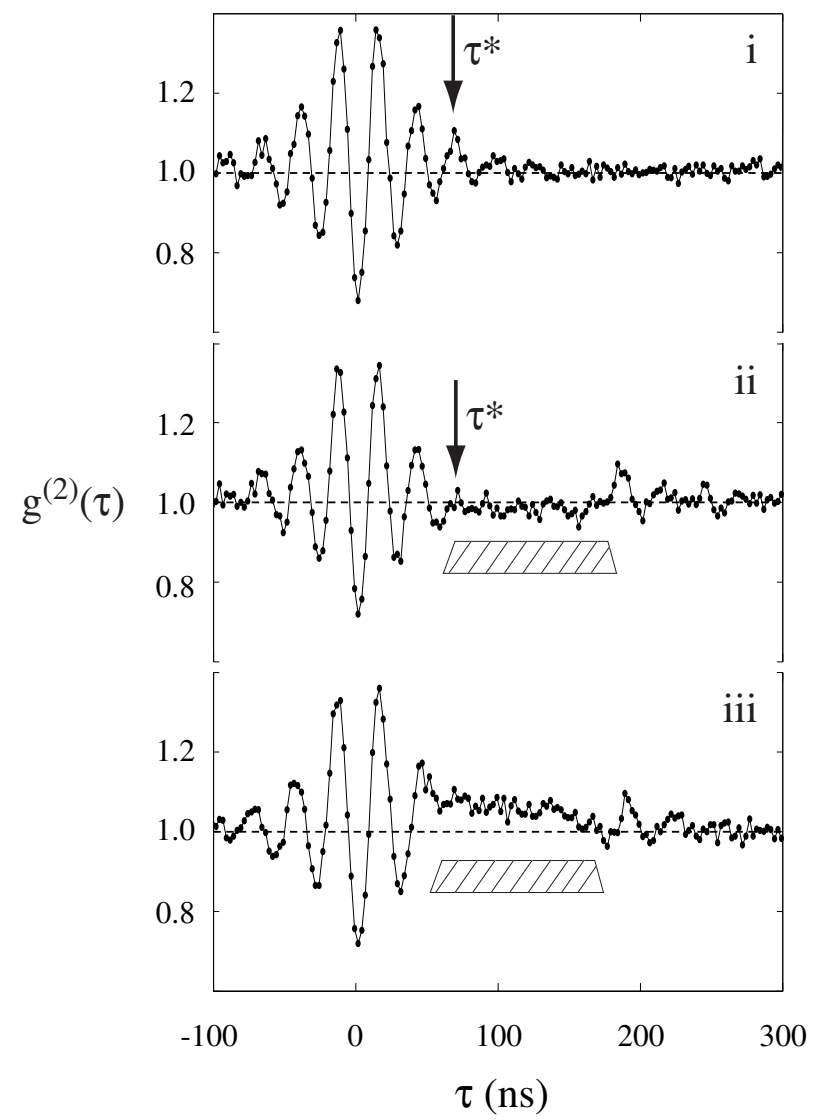

FIG. 3. Measured intensity correlation function with the feedback step applied during the shaded region: i) no feedback $(g \sqrt{N} / 2 \pi=37 \mathrm{MHz})$, ii) suppression with a step down change of $2.6 \%(g \sqrt{N} / 2 \pi=37 \mathrm{MHz})$ iii $)$ suppression with a step up change of $3.9 \%(g \sqrt{N} / 2 \pi=31 \mathrm{MHz})$. The oscillation of the system continues with the same phase and amplitude once the step is off. Note that the time $T$ for the beginning of the feedback in ii and iii is different as indicated by the position of the shaded region. The data has been binned in 2.5 ns points joined with a line.

Reversing the sign of the step produces an enhancement of the oscillations. If the time $T$ for the application of the pulse is not correct, it is not possible to achieve good suppression. There is qualitative agreement between the traces i and ii with those of the theory in Fig. 1. They show the suppression and the delayed return of the oscillation by the application of a feedback pulse to the driving intensity. Although the theoretical model does not include all the experimental details that give rise to broadening the main features of the response are clearly explained. 


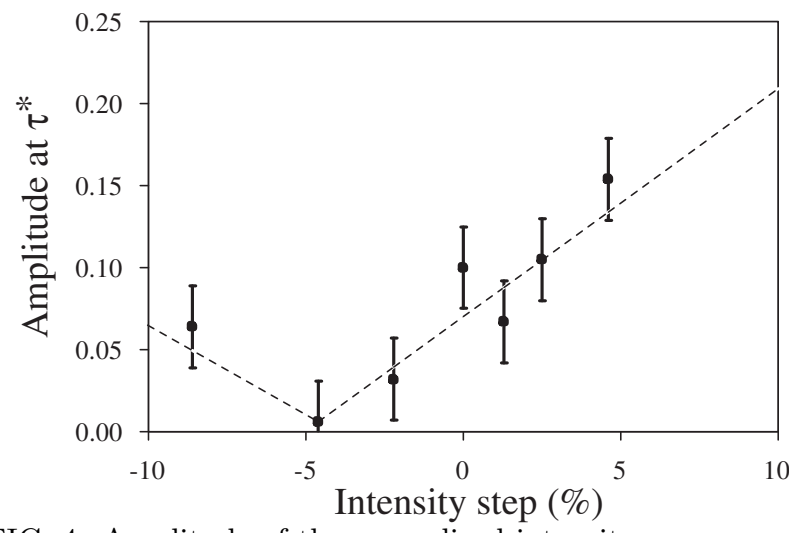

FIG. 4. Amplitude of the normalized intensity response at time $\tau *$ (first oscillation extreme of $g^{(2)}(\tau)$ after the application of the feedback pulse) as a function of the size the feedback step. The dashed line is a theoretical prediction.

We have followed the size of the amplitude of the oscillation immediately after we apply the feedback pulse, at the time $\tau^{*}$ defined in Fig. 3, to make a quantitative comparison with theory. Fig. 1 shows the results for a series of measurements that include steps up (positive) and steps down (negative). The theory (dashed line) incorporates the measured shape of the pulse (at the point $-4.6 \%$ ), all sources of dephasing present in the system are modelled by the polarization decay rate $\gamma^{\prime} / 2 \pi=9.1$ MHz. The plot shows both enhancement and suppression with quantitative agreement.

The quantum feedback in this system is triggered by a fluctuation (detection of a photon) that is large enough to significantly modify the system, because of the strong coupling. This detection prepares the system in an evolving conditional state. We then change the drive of the system and are able to freeze its evolution to a new timeindependent steady state. The suppressed oscillations return once the pulse turns off, with the same phase and amplitude information. This sort of quantum feedback is a novel way to manipulate the fragile conditional states that come from strongly coupled systems.

We would like to thank J. Gripp and J. Wang for their interest and help with this project. This work has been supported by the NSF, NIST, DAAD, and the Australian Research Council.

[1] Y. Yamamoto, N. Imoto and S. Machida, Phys. Rev. A 33, 3243 (1986).

[2] H. A. Haus and Y. Yamamoto, Phys. Rev. A 34, 270 (1986).

[3] J. H. Shapiro, G. Saplakoglu, S.-T. Ho, P. Kumar, B. E. A. Saleh, M. C. Teich, J. Opt. Soc. Am. B 4, 1604 (1987).
[4] H. M. Wiseman and G. J. Milburn, Phys. Rev. Lett. 70, 548 (1993).

[5] H. M. Wiseman, Phys. Rev. A 49, 2133 (1994).

[6] H.J. Carmichael, An Open Systems Approach to Quantum Optics (Springer-Verlag, Berlin, 1993).

[7] R. Dum, P. Zoller and H. Ritsch, Phys. Rev. A 45, 4879 (1992).

[8] K. Mølmer, Y. Castin, and J. Dalibard, J. Opt. Soc. Am. B 10, 524 (1993).

[9] A.C. Doherty and K. Jacobs, Phys. Rev. A 60, 2700 (1999).

[10] A. C. Doherty, S. Habib, K. Jacobs, H. Mabuchi, S. M. Tan, Phys. Rev. A 62, 012105 (2000).

[11] J. G. Walker and E. Jakeman, Optica-Acta. 32, 1303 (1985)

[12] P. R. Tapster, J. G. Rarity and J. S. Satchell, Phys. Rev. A 37, 2963 (1988).

[13] J. Mertz, A. Heidmann and C. Fabre, Phys. Rev. A 44, 3329 (1991).

[14] M. S. Taubman, H. Wiseman, D. E. McClelland, H.-A. Bachor, J. Opt. Soc. Am. B 12, 1792 (1995).

[15] B. C. Buchler, M. B. Gray, D. A. Shaddock, T. C. Ralph, D. E. McClelland, Opt. Lett. 24259 (1999).

[16] An exception is the following, which is an experiment of a quite different nature: R. J. Nelson, Y. Weinstein, D. Cory, and S. Lloyd, Phys. Rev. Lett. 85, 3045 (2000).

[17] H. J. Carmichael, H. Castro Beltran, G. T. Foster, and L. A. Orozco, Phys. Rev. Lett. 85, 1855 (2000).

[18] G. T. Foster, L. A. Orozco, H. M. Castro-Beltran, and H. J. Carmichael, Phys. Rev. Lett. 85, 3149 (2000).

[19] P. Berman, ed., Cavity Quantum Electrodynamics, Supplement 2 of Advances in Atomic, Molecular and Optical Physics series (Academic Press, Boston, 1994).

[20] A. Rauschenbeutel, G. Nogues, S. Osnaghi, P. Bertet, M. Brune, J. M. Raimond, and S. Haroche, Science 288, 2024 (2000).

[21] B. T. H. Varcoe, S. Brattke, M. Weidinger, and H. Walther, Nature 403, 743 (2000).

[22] C. J. Hood, R. W. Lynn, A. C. Doherty, A. S. Parkins, and H. J. Kimble, Science 287, 1447 (2000); A. C. Doherty, T. W. Lynn, C. J. Hood, and H. J. Kimble, Phys. Rev. A 63, 013401 (2001).

[23] P. W. H. Pinkse, T. Fischer, P. Maunz, and G. Rempe, Nature 404, 365 (2000).

[24] H. J. Carmichael, R. J. Brecha, and P. R. Rice, Opt. Comm. 82, 73 (1991).

[25] J. E. Reiner, W. P. Smith, L. A. Orozco, H. J. Carmichael, and P. R. Rice, J. Opt. Soc. Am. B 18, 1911 (2001).

[26] L. Mandel, E. Wolf, Optical Coherence and Quantum Optics, (Cambridge University Press, New York, 1995).

[27] G. T. Foster, S. L. Mielke, and L. A. Orozco, Phys. Rev. A 61, 53821 (2000).

[28] G. Rempe, R. J. Thompson, R. J. Brecha, W. D. Lee, and H. J. Kimble, Phys. Rev. Lett. 67, 1727 (1991).

[29] H. J. Carmichael, B. C. Sanders, Phys. Rev. A 60, 2497 (1999). 